\title{
Generic Quantum Markov Semigroups: the Fock Case
}

\author{
L. Accardi \\ Centro Vito Volterra, Facoltà di Economia \\ Università di Roma Tor Vergata \\ Via di Tor Vergata, 00133 Roma, Italy \\ e-mail: accardi@volterra.mat.uniroma2.it \\ S. Hachicha and H. Ouerdiane \\ University of Tunis-El Manar, Faculty of Sciences \\ Department of Mathematics, 1060 Tunis, Tunisia \\ e-mail: skander.hachicha@ipeit.rnu.tn \\ e-mail: habib.ouerdiane@fst.rnu.tn
}

(Received: May 31, 2005)

\begin{abstract}
We introduce the class of generic quantum Markov semigroups. Within this class we study the class corresponding to the Fock case which is further split into four sub-classes each of which contains both bounded and unbounded generators, depending on some global characteristics of the intensities of jumps. For the first two of these classes we find an explicit solution which reduces the problem of finding the quantum semigroup to the calculation of two classical semigroups, one of which is diagonal (in suitable basis) and the other one is triangular (in the same basis). In the bounded case our formula gives the unique solution. In the unbounded case it gives one solution, which we conjecture to be the minimal one.
\end{abstract}

\section{Introduction}

On quantum Markov semigroups and their generators many deep results are available and we refer to the monograph [6] or to the extensive survey article [9] for updated overviews as well as for a bibliography and $[5,10,4]$ for more recent results. However, often one is interested in particular physical models, and one needs more detailed information which not always are available from the general theory.

In particular, the class of generators for which an explicit solution of the associated master equation is available, is at the moment rather narrow. On the other hand the stochastic limit of quantum theory, when applied to quantum interacting particles, shows that the simplest class of generators corresponds to the so called "generic system", introduced in [1]. In the present paper we begin the investigation of this class and we prove that, at least in the Fock case (plus an additional "non explosive" condition) it is possible to deduce a fairly explicit formula which reduces the solution of the corresponding master equation to the solution of a classical master equation. This implies a significant reduction of the complexity of the problem. For example, in the finite dimensional case, this reduces the problem 
from an $n^{2} \times n^{2}$ matrix to an $n \times n$ one, which is a huge reduction for numerical calculations. The main result of this note is Theorem 6 .

\section{Classes of Markov Semigroups}

If we neglect the Hamiltonian part, the Markov semigroup obtained in the stochastic limit of a discrete system with generic free Hamiltonian $H_{S}$ acting on a (separable) Hilbert space $\mathcal{H}_{S}$ and interacting with a mean zero, gauge invariant Gaussian field through an interaction Hamiltonian of dipole type $H_{I}=D \otimes A_{g}^{+}+D^{+} \otimes A_{g}$, cf. $[1, \mathrm{p} .36,(1.1 .99)]$ is:

$$
\begin{aligned}
L_{\mathrm{Re}}(x)= & \sum_{\varepsilon_{\sigma^{\prime}}<\varepsilon_{\sigma}}\left[\gamma_{\sigma \sigma^{\prime}}^{-}\left(|\sigma\rangle\langle\sigma|\left\langle\sigma^{\prime}, x \sigma^{\prime}\right\rangle-\frac{1}{2}\{x,|\sigma\rangle\langle\sigma|\}\right)\right. \\
& \left.+\gamma_{\sigma \sigma^{\prime}}^{+}\left(\left|\sigma^{\prime}\right\rangle\left\langle\sigma^{\prime}\right|\langle\sigma, x \sigma\rangle-\frac{1}{2}\left\{x,\left|\sigma^{\prime}\right\rangle\left\langle\sigma^{\prime}\right|\right\}\right)\right], \quad x \in \mathcal{B}\left(\mathcal{H}_{\mathcal{S}}\right),
\end{aligned}
$$

where the suffix Re, in $L_{\text {Re }}$, means that we are neglecting the Hamiltonian term in the generator; $(|\sigma\rangle)$ is an orthonormal basis of $\mathcal{H}_{S}$

$$
\left\langle\sigma, \sigma^{\prime}\right\rangle=\delta_{\sigma \sigma^{\prime}}, \quad \sum_{\sigma}|\sigma\rangle\langle\sigma|=1
$$

whose elements are the eigenvectors of the free Hamiltonian $\left(H_{S}=\sum_{\sigma} \varepsilon_{\sigma}|\sigma\rangle\langle\sigma|\right)$. The genericity of $H_{S}$ implies that it has nondegenerate pure point spectrum. In the following, to keep a bridge with physical intuition, it will be convenient to suppose that the vectors $|\sigma\rangle$ are labelled by the corresponding energies $\varepsilon_{\sigma}$ rather than by the sequence of natural numbers.

Once fixed the basis $|\sigma\rangle$, the generator $L_{\text {Re }}$ is uniquely determined by the coefficients $\gamma_{\sigma \sigma^{\prime}}^{ \pm}$, which encode the whole physical information on the system $S$. They are positive real numbers such that

$$
\gamma_{\sigma \sigma^{\prime}}^{ \pm}=0 \quad \text { if } \quad \varepsilon_{\sigma} \leq \varepsilon_{\sigma^{\prime}}
$$

(in particular they are zero on the diagonal) and which represent the real part of the "generalized susceptibilities" whose structure, deduced through stochastic limit techniques, is described in [3, p.19-21]. Physically $\gamma_{\sigma \sigma^{\prime}}^{+}$represents the rate of jumps "up" $\left(\varepsilon_{\sigma^{\prime}} \uparrow \varepsilon_{\sigma}\right)$ and $\gamma_{\sigma \sigma^{\prime}}^{-}$represents the rate of jumps "down" $\left(\varepsilon_{\sigma} \downarrow \varepsilon_{\sigma^{\prime}}\right)$. Let us introduce the notations

$$
\begin{aligned}
|\sigma\rangle\langle\sigma|=: & P_{\sigma}, \\
\langle\sigma, x \sigma\rangle & =: \quad \sigma(x) .
\end{aligned}
$$

Then

$$
\begin{aligned}
P_{\sigma} P_{\sigma^{\prime}} & =\delta_{\sigma \sigma^{\prime}} P_{\sigma}, \quad \sum_{\sigma} P_{\sigma}=1, \\
\sigma^{\prime}\left(x P_{\sigma}\right) & =\sigma^{\prime}\left(P_{\sigma} x\right)=\delta_{\sigma \sigma^{\prime}} \sigma^{\prime}(x) .
\end{aligned}
$$


In these notations the generator (1) becomes

$$
L_{\operatorname{Re}}(x)=\sum_{\varepsilon_{\sigma^{\prime}}<\varepsilon_{\sigma}}\left[\gamma_{\sigma \sigma^{\prime}}^{-}\left(P_{\sigma} \sigma^{\prime}(x)-\frac{1}{2}\left\{x, P_{\sigma}\right\}\right)+\gamma_{\sigma \sigma^{\prime}}^{+}\left(P_{\sigma^{\prime}} \sigma(x)-\frac{1}{2}\left\{x, P_{\sigma^{\prime}}\right\}\right)\right] .
$$

Our goal is to study various classes of these semigroups and to classify them according to the properties of the coefficients $\gamma_{\sigma \sigma^{\prime}}^{ \pm}$.

In the following we will omit the suffix $R e$, in $L_{\mathrm{Re}}$, because throughout the present paper we consider only this case.

\section{The Simplest Class of Generators}

The simplest class of Markovian generators is obtained when there are no jumps up:

$$
\gamma_{\sigma \sigma^{\prime}}^{+}=0, \quad \forall \sigma, \sigma^{\prime}
$$

and there is a single (nonzero) jump down (2-level system):

$$
D=\left|\sigma_{0}\right\rangle\left\langle\sigma_{0}^{\prime}\right|, \quad \varepsilon_{\sigma_{0}^{\prime}}<\varepsilon_{\sigma_{0}} .
$$

In this case

$$
\left|\sigma_{0}\right\rangle \perp\left\langle\sigma_{0}^{\prime}\right|
$$

and

$$
L(x)=\gamma_{\sigma_{0} \sigma_{0}^{\prime}}^{-}\left(\left|\sigma_{0}\right\rangle\left\langle\sigma_{0}\right|\left\langle\sigma_{0}^{\prime}, x \sigma_{0}^{\prime}\right\rangle-\frac{1}{2}\left\{x,\left|\sigma_{0}\right\rangle\left\langle\sigma_{0}\right|\right\}\right) .
$$

This is an elementary semigroup about which everything is well known. The discussion that follows isolates some algebraic properties, of the various pieces which form the generator of this semigroup, which persist in the infinite dimensional case and which allow to deduce, also in this case, a formula similar to the one deduced in the present section.

LEMMA 1 Defining

$$
\begin{aligned}
& S_{1}(x):=\left|\sigma_{0}\right\rangle\left\langle\sigma_{0}\right|\left\langle\sigma_{0}^{\prime}, x \sigma_{0}^{\prime}\right\rangle=: \sigma_{0}^{\prime}(x) P_{\sigma_{0}}, \\
& S_{2}(x):=-\frac{1}{2}\left\{x, P_{\sigma_{0}}\right\}
\end{aligned}
$$

one has

$$
S_{2}\left(P_{\sigma_{0}}\right)=-P_{\sigma_{0}}
$$

and, since $\sigma_{0} \perp \sigma_{0}^{\prime}$, then

$$
S_{1}\left(P_{\sigma_{0}}\right)=0
$$

so that in particular (16) holds

$$
S_{1}^{2}=0 \text {. }
$$

Conditions (15) and (14) imply the following commutation relations:

$$
\begin{aligned}
& S_{1} S_{2}=0, \\
& S_{2} S_{1}=-S_{1}
\end{aligned}
$$


Proof. In fact, from (12) and (13) it follows that

$$
\begin{aligned}
S_{1} S_{2}(x) & =S_{1}\left(-\frac{1}{2}\left\{x, P_{\sigma_{0}}\right\}\right)=-\frac{1}{2}\left(S_{1}\left(x P_{\sigma_{0}}\right)+S_{1}\left(P_{\sigma_{0}} x\right)\right) \\
& =-\frac{1}{2}\left|\sigma_{0}\right\rangle\left\langle\sigma_{0}\right|\left(\left\langle\sigma_{0}^{\prime}, x P_{\sigma_{0}} \sigma_{0}^{\prime}\right\rangle+\left\langle\sigma_{0}^{\prime}, P_{\sigma_{0}} x \sigma_{0}^{\prime}\right\rangle\right)=0 \\
S_{2} S_{1}(x) & =\sigma_{0}^{\prime}(x) S_{2}\left(P_{\sigma_{0}}\right)=-\sigma_{0}^{\prime}(x) P_{\sigma_{0}}=-S_{1}(x)
\end{aligned}
$$

and this proves the statement.

\section{THEOREM 1 It holds}

$$
e^{t\left(S_{1}+S_{2}\right)}=e^{t S_{2}}+\left(1-e^{-t}\right) S_{1} .
$$

Proof. Given the relations (17) (18), (16), the identity (19) follows from the identity

$$
\left(S_{1}+S_{2}\right)\left(e^{t S_{2}}+\left(1-e^{-t}\right) S_{1}\right)=S_{2} e^{t S_{2}}+e^{-t} S_{1},
$$

which is the derivative of the right hand side of (19). So the two sides of (19) satisfy the same ordinary differential equation with the same initial condition, hence they coincide.

COROLLARY 2 The semigroup generated by

$$
L^{-}(x):=\gamma_{\sigma_{0} \sigma_{0}^{\prime}}^{-}\left(P_{\sigma_{0}} \sigma_{0}^{\prime}(x)-\frac{1}{2}\left\{P_{\sigma_{0}}, x\right\}\right)=\gamma_{\sigma_{0} \sigma_{0}^{\prime}}^{-}\left(S_{1}+S_{2}\right)(x)
$$

is

$$
e^{t L^{-}}(x)=\left(e^{-t \gamma_{\sigma_{0} \sigma_{0}^{\prime}}^{-} / 2} P_{\sigma_{0}}+P_{\sigma_{0}}^{\perp}\right) x\left(e^{-t \gamma_{\sigma_{0}, \sigma_{0}^{\prime}}^{-} / 2} P_{\sigma_{0}}+P_{\sigma_{0}}^{\perp}\right)+\left(1-e^{-t}\right) \sigma_{0}^{\prime}(x) P_{\sigma_{0}} .
$$

Proof. From Theorem 1, with the time rescaling $t \rightarrow \gamma_{\sigma_{0} \sigma_{0}^{\prime}}^{-} t$ we can restrict our consideration to the semigroup

$$
e^{-\frac{t}{2} P_{\sigma_{0}}} x e^{-\frac{t}{2} P_{\sigma_{0}}}=P_{2}^{t}(x) .
$$

Its generator is

$$
\left.\partial_{t}\right|_{t=0} P_{2}^{t}(x)=-\frac{1}{2} P_{\sigma_{0}} x-x \frac{1}{2} P_{\sigma_{0}}=S_{2}(x) .
$$

Thus

$$
e^{-t S_{2}}(x)=e^{-\frac{t}{2}\left\{P_{\sigma_{0}} \cdot \cdot\right\}}(x)=e^{-\frac{t}{2} P_{\sigma_{0}}} x e^{-\frac{t}{2} P_{\sigma_{0}}} .
$$

But $P_{\sigma_{0}}=\left|\sigma_{0}\right\rangle\left\langle\sigma_{0}\right|$ is a self-adjoint projection. Therefore

$$
e^{-\frac{t}{2} P_{\sigma_{0}}}=e^{-t / 2} P_{\sigma_{0}}+P_{\sigma_{0}}^{\perp}
$$

Hence

$$
e^{-\frac{t}{2}\left\{P_{\sigma_{0}} \cdot \cdot\right\}}(x)=\left(e^{-t / 2} P_{\sigma_{0}}+P_{\sigma_{0}}^{\perp}\right) x\left(e^{-t / 2} P_{\sigma_{0}}+P_{\sigma_{0}}^{\perp}\right)
$$

from which and (12) the statement follows. 


\section{Generators with $\gamma^{+}=0$}

In this section we begin to study the class of generators of the form (7), (2) under the additional condition (8). These generators will be called generic Fock generators because, in the stochastic limit, they arise from interactions of systems with a generic free Hamiltonian $H_{S}$ (cf. [1]) and with fields in the Fock state (i.e when the Gaussian field, mentioned in the beginning of Sect. 2., is a Fock field). Such generators have the form

$$
L^{-}(x)=\sum_{\varepsilon_{\sigma^{\prime}}<\varepsilon_{\sigma}} \gamma_{\sigma \sigma^{\prime}}^{-}\left(P_{\sigma} \sigma^{\prime}(x)-\frac{1}{2}\left\{x, P_{\sigma}\right\}\right) .
$$

In the following we will frequently use the following simple remark:

LEMMA 2 Suppose that $A B=B A$. Then

$$
\begin{aligned}
\{B,\{A, \cdot\}\} & =\{A,\{B, \cdot\}\} \\
{[B,[A, \cdot]] } & =[A,[B, \cdot]] .
\end{aligned}
$$

Proof.

$$
\begin{aligned}
\{B,\{A, x\}\} & =\{B, A x\}+\{B, x A\}=B A x+A x B+B x A+x A B \\
& =A B x+B x A+A x B+x B A=\{A, B x\}+\{A, x B\} \\
& =\{A,\{B, x\}\}
\end{aligned}
$$

and similarly for the commutator.

The following lemma is applicable to a slightly larger class of generators than those considered here.

LEMMA 3 In the notations (5), (6) define $\forall \sigma, \sigma^{\prime}$

$$
\begin{aligned}
S_{\sigma, \sigma^{\prime}}(x) & :=P_{\sigma} \sigma^{\prime}(x), \\
S_{\sigma}(x) & :=-\frac{1}{2}\left\{x, P_{\sigma}\right\} .
\end{aligned}
$$

Then $\forall \sigma, \sigma^{\prime}, \tau, \tau^{\prime}$

$$
\begin{aligned}
S_{\sigma, \sigma^{\prime}} S_{\tau} & =-\delta_{\sigma^{\prime}, \tau} S_{\sigma, \sigma^{\prime}} \\
S_{\tau} S_{\sigma, \sigma^{\prime}} & =-\delta_{\sigma, \tau} S_{\sigma, \sigma^{\prime}} \\
S_{\sigma} S_{\sigma^{\prime}} & =S_{\sigma^{\prime}} S_{\sigma} \\
S_{\sigma, \sigma^{\prime}} S_{\tau, \tau^{\prime}} & =\delta_{\sigma^{\prime}, \tau} S_{\sigma, \tau^{\prime}}
\end{aligned}
$$

Proof.

$$
\begin{aligned}
S_{\sigma \sigma^{\prime}} S_{\tau}(x) & =P_{\sigma} \sigma^{\prime}\left(S_{\tau}(x)\right)=-\frac{1}{2} P_{\sigma} \sigma^{\prime}\left(x P_{\tau}\right)-\frac{1}{2} P_{\sigma} \sigma^{\prime}\left(P_{\tau} x\right) \\
& =-\frac{1}{2} P_{\sigma} \delta_{\sigma^{\prime} \tau} \sigma^{\prime}(x)-\frac{1}{2} P_{\sigma} \delta_{\sigma^{\prime} \tau} \sigma^{\prime}(x)=-\delta_{\sigma^{\prime} \tau} P_{\sigma} \sigma^{\prime}(x) \\
& =-\delta_{\sigma^{\prime} \tau} S_{\sigma \sigma^{\prime}}(x)
\end{aligned}
$$


and this proves (25).

$$
\begin{aligned}
S_{\tau} S_{\sigma \sigma^{\prime}}(x) & =\sigma^{\prime}(x) S_{\tau}\left(P_{\sigma}\right)=\sigma^{\prime}(x)\left(-\frac{1}{2}\left\{P_{\tau}, P_{\sigma}\right\}\right) \\
& =-\sigma^{\prime}(x) P_{\sigma} \delta_{\tau \sigma}=-\delta_{\tau \sigma} S_{\sigma \sigma^{\prime}}(x)
\end{aligned}
$$

and this proves (26). (27) follows from Lemma 2. Finally,

$$
\begin{aligned}
S_{\sigma \sigma^{\prime}} S_{\tau \tau^{\prime}}(x) & =\tau^{\prime}(x) S_{\sigma \sigma^{\prime}}\left(P_{\tau}\right)=\tau^{\prime}(x) \sigma^{\prime}\left(P_{\tau}\right) P_{\sigma} \\
& =\tau^{\prime}(x) \delta_{\sigma^{\prime} \tau} P_{\sigma}=\delta_{\sigma^{\prime} \tau} S_{\sigma \tau^{\prime}}(x)
\end{aligned}
$$

and this proves (28).

In the notations $(23),(24)$ the generator (22) takes the form

$$
L^{-}:=\sum_{\varepsilon_{\sigma^{\prime}}<\varepsilon_{\sigma}} \gamma_{\sigma \sigma^{\prime}}^{-}\left(S_{\sigma \sigma^{\prime}}+S_{\sigma}\right)=: \sum_{\varepsilon_{\sigma^{\prime}}<\varepsilon_{\sigma}} L_{\sigma \sigma^{\prime}}^{-} .
$$

In general the $L_{\sigma \sigma^{\prime}}^{-}$will not commute among themselves.

Let us now introduce the notation

$$
\lambda_{\sigma^{\prime}}^{-}:=\sum_{\left\{\sigma: \varepsilon_{\sigma^{\prime}}<\varepsilon_{\sigma}\right\}} \gamma_{\sigma \sigma^{\prime}}^{-} .
$$

Notice that the $\gamma_{\sigma \sigma^{\prime}}^{-}$are positive. Therefore

$$
\begin{gathered}
\forall \sigma \quad \lambda_{\sigma^{\prime}}^{-} \geq \gamma_{\sigma \sigma^{\prime}}^{-}, \\
\forall \sigma \quad \lambda_{\sigma^{\prime}}^{-}=0 \quad \Longleftrightarrow \gamma_{\sigma \sigma^{\prime}}^{-}=0 .
\end{gathered}
$$

We distinguish 4 cases:

$$
\begin{array}{rll}
\forall \sigma \quad 0<\lambda_{\sigma}^{-}<+\infty \\
\exists \sigma \quad \lambda_{\sigma}^{-}=0 ; & \forall \sigma \quad \lambda_{\sigma}^{-}<+\infty \\
\exists \sigma \quad \lambda_{\sigma}^{-}=+\infty ; & \forall \sigma & \lambda_{\sigma}^{-}>0 \\
\exists \sigma \quad \lambda_{\sigma}^{-}=0 ; & \exists \tau & \lambda_{\tau}^{-}=+\infty .
\end{array}
$$

With the condition (31) the generator $L^{-}$can be written in the GKSL (GoriniKossakowski-Sudarshan-Lindblad) form:

$$
L^{-}(x)=-G x+\sum_{\varepsilon_{\sigma^{\prime}}<\varepsilon_{\sigma}} V_{\sigma \sigma^{\prime}}^{*} x V_{\sigma \sigma^{\prime}}-x G,
$$

where $G=\sum_{\sigma^{\prime}} \lambda_{\sigma^{\prime}}^{-}\left|\sigma^{\prime}\right\rangle\left\langle\sigma^{\prime}\right|$ and $V_{\sigma \sigma^{\prime}}=\sqrt{\gamma_{\sigma \sigma^{\prime}}^{-}}\left|\sigma^{\prime}\right\rangle\langle\sigma|$. Here $G$ is self-adjoint and the $V_{\sigma \sigma^{\prime}}$ are bounded. Therefore the general method of the minimal semigroup (see $[7,8]$ ) can be applied. Our method instead will use the special structure of the present class of generators to deduce an explicit formula. 
Remark 1 If in the expression

$$
\sum_{\sigma^{\prime}} \lambda_{\sigma^{\prime}}^{-}\left[\sum_{\left\{\sigma: \varepsilon_{\sigma^{\prime}}<\varepsilon_{\sigma}\right\}}\left(\frac{\gamma_{\sigma \sigma^{\prime}}^{-}}{\lambda_{\sigma^{\prime}}^{-}} S_{\sigma \sigma^{\prime}}+S_{\sigma^{\prime}}\right)\right]
$$

we introduce the stochastic matrix

$$
\frac{\gamma_{\sigma \sigma^{\prime}}^{-}}{\lambda_{\sigma^{\prime}}^{-}}=: p_{\sigma \sigma^{\prime}}^{-}, \quad p_{\sigma \sigma^{\prime}}^{-}=0 \quad \text { if } \quad \lambda_{\sigma}^{-},=0, \quad p_{\sigma \sigma^{\prime}}^{-} \geq 0, \quad \sum_{\sigma} p_{\sigma \sigma^{\prime}}^{-}=1
$$

then the generator (29) takes the form

$$
\sum_{\sigma^{\prime}} \lambda_{\sigma^{\prime}}^{-} \sum_{\left\{\sigma: \varepsilon_{\sigma^{\prime}}<\varepsilon_{\sigma}\right\}}\left(p_{\sigma \sigma^{\prime}}^{-} S_{\sigma \sigma^{\prime}}+S_{\sigma^{\prime}}\right) .
$$

5. The Class: $\gamma^{+}=0,0<\lambda^{-}<\infty$

In the notations of Sect. 4 we begin to study those Markovian generators which satisfy conditions (8) and (31). Under these conditions the generator (29) takes the form

$$
L^{-}=\sum_{\varepsilon_{\sigma^{\prime}}<\varepsilon_{\sigma}} \gamma_{\sigma \sigma^{\prime}}^{-} S_{\sigma \sigma^{\prime}}+\sum_{\sigma}\left(\sum_{\varepsilon_{\sigma^{\prime}}<\varepsilon_{\sigma}} \gamma_{\sigma \sigma^{\prime}}^{-}\right) S_{\sigma}=\sum_{\varepsilon_{\sigma^{\prime}}<\varepsilon_{\sigma}} \gamma_{\sigma \sigma^{\prime}}^{-} S_{\sigma \sigma^{\prime}}+\sum_{\sigma} \lambda_{\sigma}^{-} S_{\sigma} .
$$

For any matrix

$$
x=\left(x_{\sigma \sigma^{\prime}}\right)
$$

let us introduce the notation

$$
S_{1}(x):=\sum_{\sigma, \sigma^{\prime}} x_{\sigma \sigma^{\prime}} S_{\sigma \sigma^{\prime}}
$$

and for any diagonal matrix

$$
\lambda=\left(\delta_{\sigma \sigma^{\prime}} \lambda_{\sigma^{\prime}}\right)
$$

we denote

$$
S_{2}(\lambda):=\sum_{\sigma} \lambda_{\sigma} S_{\sigma} .
$$

With these notations, the generator $L^{-}$can be written as

$$
L^{-}=S_{1}\left(\gamma^{-}\right)+S_{2}\left(\lambda^{-}\right),
$$

where $\gamma^{-}$denotes the triangular matrix

$$
\left(\gamma^{-}\right)_{\sigma, \sigma^{\prime}}:=\left\{\begin{array}{cl}
\gamma_{\sigma \sigma^{\prime}}^{-} & \text {if } \varepsilon_{\sigma^{\prime}}<\varepsilon_{\sigma} \\
0 & \text { otherwise }
\end{array}\right.
$$

and $\lambda^{-}$denotes the diagonal matrix

$$
\left(\lambda^{-}\right)_{\sigma^{\prime} \tau}=\delta_{\sigma^{\prime} \tau} \lambda_{\sigma^{\prime}}^{-} .
$$


LEMMA 4 Both maps

$$
x \mapsto S_{1}(x), \quad \lambda \mapsto S_{2}(\lambda)
$$

are linear and continuous in norm. $S_{1}(\cdot)$ is multiplicative and the range of $S_{2}(\cdot)$ is abelian.

Proof. Given two matrices $x, x^{\prime}$ one has, using (28)

$$
\begin{aligned}
S_{1}(x) S_{1}\left(x^{\prime}\right) & =\sum_{\sigma, \sigma^{\prime}} \sum_{\tau, \tau^{\prime}} x_{\sigma \sigma^{\prime}} x_{\tau \tau^{\prime}}^{\prime} S_{\sigma \sigma^{\prime}} S_{\tau \tau^{\prime}}=\sum_{\sigma, \sigma^{\prime}, \tau, \tau^{\prime}} x_{\sigma \sigma^{\prime}} x_{\tau \tau^{\prime}}^{\prime} \delta_{\sigma^{\prime} \tau} S_{\sigma, \tau^{\prime}} \\
& =\sum_{\sigma, \tau^{\prime}}\left(\sum_{\sigma^{\prime}} x_{\sigma \sigma^{\prime}} x_{\sigma^{\prime} \tau^{\prime}}^{\prime}\right) S_{\sigma \tau^{\prime}}=S_{1}\left(x x^{\prime}\right) .
\end{aligned}
$$

Therefore $S_{1}$ is multiplicative.

If $\lambda, \lambda^{\prime}$ are diagonal matrices, then using (27)

$$
S_{2}(\lambda) S_{2}\left(\lambda^{\prime}\right)=\sum_{\sigma, \sigma^{\prime}} \lambda_{\sigma} \lambda_{\sigma^{\prime}}^{\prime} S_{\sigma} S_{\sigma^{\prime}}=\sum_{\sigma^{\prime}, \sigma} \lambda_{\sigma^{\prime}}^{\prime} \lambda_{\sigma} S_{\sigma^{\prime}} S_{\sigma}=S_{2}\left(\lambda^{\prime}\right) S_{2}(\lambda) .
$$

Since linearity is obvious in both cases, this ends the proof.

LEMMA 5 In the notations (36), (37), for any $m, n \in \mathbb{N}$ and $s, t \in \mathbb{R}$, one has:

$$
\begin{aligned}
S_{1}\left(\gamma^{-}\right)^{m} S_{2}\left(\lambda^{-}\right)^{n} & =(-1)^{n} S_{1}\left(\gamma^{-m} \lambda^{-n}\right), \\
S_{2}\left(\lambda^{-}\right)^{m} S_{1}\left(\gamma^{-}\right)^{n} & =(-1)^{m} S_{1}\left(\lambda^{-m} \gamma^{-n}\right), \\
S_{1}\left(\gamma^{-}\right) e^{t S_{2}\left(\lambda^{-}\right)} & =S_{1}\left(\gamma^{-} e^{-t \lambda^{-}}\right), \\
e^{s S_{1}\left(\gamma^{-}\right)} e^{t S_{2}\left(\lambda^{-}\right)} & =S_{1}\left(e^{s \gamma^{-}} e^{-t \lambda^{-}}\right), \\
e^{s S_{2}\left(\lambda^{-}\right)} e^{t S_{1}\left(\gamma^{-}\right)} & =S_{1}\left(e^{-s \lambda^{-}} e^{t \gamma^{-}}\right) .
\end{aligned}
$$

Proof. Let us observe that

$$
\begin{aligned}
S_{1}\left(\gamma^{-}\right) S_{2}\left(\lambda^{-}\right) & =\sum_{\sigma, \sigma^{\prime}} \sum_{\tau} \gamma_{\sigma \sigma^{\prime}}^{-} \lambda_{\tau}^{-} S_{\sigma \sigma^{\prime}} S_{\tau}=-\sum_{\sigma, \sigma^{\prime}} \sum_{\tau} \gamma_{\sigma \sigma^{\prime}}^{-} \lambda_{\tau}^{-} \delta_{\sigma^{\prime} \tau} S_{\sigma \sigma^{\prime}} \\
& =-\sum_{\sigma, \sigma^{\prime}} \gamma_{\sigma \sigma^{\prime}}^{-} \lambda_{\sigma^{\prime}}^{-} S_{\sigma \sigma^{\prime}}=-S_{1}\left(\gamma^{-} \lambda^{-}\right), \\
S_{2}\left(\lambda^{-}\right) S_{1}\left(\gamma^{-}\right) & =\sum_{\tau} \sum_{\sigma, \sigma^{\prime}} \lambda_{\tau}^{-} \gamma_{\sigma \sigma^{\prime}}^{-} S_{\tau} S_{\sigma \sigma^{\prime}}=-\sum_{\tau} \sum_{\sigma, \sigma^{\prime}} \lambda_{\tau}^{-} \gamma_{\sigma \sigma^{\prime}}^{-} \delta_{\sigma \tau} S_{\sigma \sigma^{\prime}} \\
& =-\sum_{\sigma, \sigma^{\prime}} \lambda_{\sigma}^{-} \gamma_{\sigma \sigma^{\prime}}^{-} S_{\sigma \sigma^{\prime}}=-S_{1}\left(\lambda^{-} \gamma^{-}\right) .
\end{aligned}
$$

By induction from (45)

$$
S_{2}\left(\lambda^{-}\right)^{n} S_{1}\left(\gamma^{-}\right)=(-1)^{n} S_{1}\left(\lambda^{-n} \gamma^{-}\right) .
$$

Hence, by the linearity and norm continuity of the maps (41)

$$
e^{t S_{2}\left(\lambda^{-}\right)} S_{1}\left(\gamma^{-}\right)=S_{1}\left(e^{-t \lambda^{-}} \gamma^{-}\right) .
$$


By induction from (44)

$$
S_{1}\left(\gamma^{-}\right) S_{2}\left(\lambda^{-}\right)^{n}=(-1)^{n} S_{1}\left(\gamma^{-} \lambda^{-n}\right) .
$$

By the linearity and continuity of the maps (41)

$$
S_{1}\left(\gamma^{-}\right) e^{t S_{2}\left(\lambda^{-}\right)}=S_{1}\left(\gamma^{-} e^{-t \lambda^{-}}\right) .
$$

Combining (46), (47) and the multiplicative property of $S_{1}$, one obtains

$$
\begin{aligned}
S_{2}\left(\lambda^{-}\right)^{n} S_{1}\left(\gamma^{-}\right)^{m} & =S_{2}\left(\lambda^{-}\right)^{n} S_{1}\left(\gamma^{-}\right) S_{1}\left(\gamma^{-}\right)^{m-1} \\
& =(-1)^{n} S_{1}\left(\lambda^{-n} \gamma^{-}\right) S_{1}\left(\gamma^{-(m-1)}\right) \\
& =(-1)^{n} S_{1}\left(\lambda^{-n} \gamma^{-m}\right) .
\end{aligned}
$$

From this one deduces that

$$
e^{s S_{2}\left(\lambda^{-}\right)} e^{t S_{1}\left(\gamma^{-}\right)}=S_{1}\left(e^{-s \lambda^{-}} e^{t \gamma^{-}}\right) .
$$

Similarly

$$
\begin{aligned}
S_{1}\left(\gamma^{-}\right)^{m} S_{2}\left(\lambda^{-}\right)^{n} & =S_{1}\left(\gamma^{-}\right)^{m-1} S_{1}\left(\gamma^{-}\right) S_{2}\left(\lambda^{-}\right)^{n} \\
& =S_{1}\left(\gamma^{-(m-1)}\right) S_{1}\left(\gamma^{-}(-1)^{n} \lambda^{-n}\right) \\
& =S_{1}\left(\gamma^{-m}(-1)^{n} \lambda^{-n}\right) .
\end{aligned}
$$

From this one deduces that

$$
e^{s S_{1}\left(\gamma^{-}\right)} e^{t S_{2}\left(\lambda^{-}\right)}=S_{1}\left(e^{s \gamma^{-}} e^{-t \lambda^{-}}\right) .
$$

LEMMA 6 For any $n \in \mathbb{N}$

$$
\left(S_{1}\left(\gamma^{-}\right)+S_{2}\left(\lambda^{-}\right)\right)^{n}=S_{2}\left(\lambda^{-}\right)^{n}-S_{1}\left(\left(-\lambda^{-}\right)^{n}\right)+S_{1}\left(\left(\gamma^{-}-\lambda^{-}\right)^{n}\right) .
$$

Proof. It is convenient to write

$$
\theta_{1}:=\gamma^{-}, \quad \theta_{2}:=\lambda^{-} .
$$

With this notation

$$
\begin{aligned}
& \left(S_{1}\left(\gamma^{-}\right)+S_{2}\left(\lambda^{-}\right)\right)^{n}=\left(\sum_{\varepsilon \in\{1,2\}} S_{\varepsilon}\left(\theta_{\varepsilon}\right)\right)^{n} \\
& =\sum_{\left\{\bar{\varepsilon}=\left(\varepsilon_{1}, \ldots, \varepsilon_{n}\right) \in\{1,2\}^{n}\right\}} S_{\varepsilon_{1}}\left(\theta_{\varepsilon_{1}}\right) \ldots S_{\varepsilon_{n}}\left(\theta_{\varepsilon_{n}}\right) \\
& =S_{2}\left(\theta_{2}\right)^{n}+\sum_{k=0}^{n-1} \sum_{\left\{\bar{\varepsilon} \in\{1,2\}^{n},\left|\left\{j: \varepsilon_{j}=2\right\}=k\right|\right\}} S_{\varepsilon_{1}}\left(\theta_{\varepsilon_{1}}\right) \ldots S_{\varepsilon_{n}}\left(\theta_{\varepsilon_{n}}\right)
\end{aligned}
$$




$$
\begin{aligned}
& =S_{2}\left(\theta_{2}\right)^{n}+\sum_{k=0}^{n-1}(-1)^{k} \sum_{\left\{\bar{\varepsilon} \in\{1,2\}^{n},\left|\left\{j: \varepsilon_{j}=2\right\}\right|=k\right\}} S_{1}\left(\theta_{\varepsilon_{1}} \ldots \theta_{\varepsilon_{n}}\right) \\
& =S_{2}\left(\theta_{2}\right)^{n}-S_{1}\left((-1)^{n} \theta_{2}^{n}\right)+S_{1}\left(\sum_{k=0}^{n}(-1)^{k} \sum_{\left\{\bar{\varepsilon} \in\{1,2\}^{n},\left|\left\{j: \varepsilon_{j}=2\right\}\right|=k\right\}} \theta_{\varepsilon_{1}} \ldots \theta_{\varepsilon_{n}}\right) \\
& =S_{2}\left(\theta_{2}\right)^{n}-S_{1}\left((-1)^{n} \theta_{2}^{n}\right)+S_{1}\left(\left(\theta_{1}-\theta_{2}\right)^{n}\right) .
\end{aligned}
$$

COROLLARY 3 It holds

$$
e^{t\left(S_{1}\left(\gamma^{-}\right)+S_{2}\left(\lambda^{-}\right)\right)}=e^{t S_{2}\left(\lambda^{-}\right)}-S_{1}\left(e^{-t \lambda^{-}}\right)+S_{1}\left(e^{t\left(\gamma^{-}-\lambda^{-}\right)}\right) .
$$

Proof. The proof follows from (48) plus the linearity and continuity of $S_{1}, S_{2}$.

LEMMA 7 For each eigenvector $|\sigma\rangle$ of $H_{S}$, define the contraction semigroup

$$
V_{\sigma}^{t}:=e^{-t \lambda_{\sigma}^{-} / 2} P_{\sigma}+P_{\sigma}^{\perp} .
$$

Then $\left\{V_{\sigma}^{t}: \sigma \in \mathrm{Sp}\left(H_{S}\right)\right\}$ is a commuting family of contraction semigroups and one has

$$
e^{t S_{2}\left(\lambda^{-}\right)}(x)=\prod_{\sigma} V_{\sigma}^{t} x V_{\sigma}^{t}=\left(\prod_{\sigma} V_{\sigma}^{t}\right) x\left(\prod_{\sigma} V_{\sigma}^{t}\right)
$$

where the product $\prod_{\sigma} V_{\sigma}^{t}$ is strongly convergent.

Proof. From the definition (37) of $S_{2}(\lambda)$ it follows that

$$
S_{2}(\lambda)=\sum_{\sigma} \lambda_{\sigma} S_{\sigma}
$$

Therefore, from (27)

$$
e^{t S_{2}(\lambda)}=e^{t \sum_{\sigma} \lambda_{\sigma} S_{\sigma}}=\prod_{\sigma} e^{t \lambda_{\sigma} S_{\sigma}}
$$

From (24) we know that

$$
S_{\sigma}(x)=-\frac{1}{2}\left\{x, P_{\sigma}\right\}
$$

so that

$$
e^{t S_{\sigma}}(x)=e^{-\frac{t}{2} P_{\sigma}} x e^{-\frac{t}{2} P_{\sigma}}=\left(e^{-t / 2} P_{\sigma}+P_{\sigma}^{\perp}\right) x\left(e^{-\frac{t}{2}} P_{\sigma}+P_{\sigma}^{\perp}\right) .
$$

From this (51) immediately follows. 
LEMMA 8 If $(|\sigma\rangle)$ is any orthonormal set, then

$$
\prod_{\sigma}\left(e^{-t \lambda_{\sigma} / 2} P_{\sigma}+P_{\sigma}^{\perp}\right)=\sum_{\sigma} e^{-t \lambda_{\sigma} / 2} P_{\sigma}+\prod_{\sigma} P_{\sigma}^{\perp} .
$$

In particular, if $(|\sigma\rangle)$ is an orthonormal basis

$$
\prod_{\sigma}\left(e^{-t \lambda_{\sigma} / 2} P_{\sigma}+P_{\sigma}^{\perp}\right)=\sum_{\sigma} e^{-t \lambda_{\sigma} / 2} P_{\sigma}
$$

Proof. To evaluate the product consider first the case of 2 factors:

$$
\left(e^{\frac{-t \lambda_{\sigma}}{2}} P_{\sigma}+P_{\sigma}^{\perp}\right)\left(e^{\frac{-t \lambda_{\tau}}{2}} P_{\tau}+P_{\tau}^{\perp}\right)=e^{\frac{-t \lambda_{\sigma}}{2}} P_{\sigma}+e^{\frac{-t \lambda_{\tau}}{2}} P_{\tau}+P_{\sigma}^{\perp} P_{\tau}^{\perp} .
$$

Suppose by induction that

$$
\prod_{j=1}^{n}\left(e^{\frac{-t \lambda \sigma_{j}}{2}} P_{\sigma_{j}}+P_{\sigma_{j}}^{\perp}\right)=\sum_{j=1}^{n} e^{\frac{-t \lambda \sigma_{j}}{2}} P_{\sigma_{j}}+\prod_{j=1}^{n} P_{\sigma_{j}}^{\perp} .
$$

Then

$$
\begin{aligned}
\prod_{j=1}^{n+1}\left(e^{\frac{-t \lambda \sigma_{j}}{2}} P_{\sigma_{j}}+P_{\sigma_{j}}^{\perp}\right) & =\left(\sum_{j=1}^{n} e^{-t \lambda_{\sigma_{j}}} P_{\sigma_{j}}+\prod_{j=1}^{n} P_{\sigma_{j}}^{\perp}\right)\left(e^{\frac{-t \lambda \sigma_{n+1}}{2}} P_{\sigma_{n+1}}+P_{\sigma_{n+1}}^{\perp}\right) \\
& =\sum_{j=1}^{n} e^{\frac{-t \lambda \sigma_{j}}{2}} P_{\sigma_{j}}+e^{\frac{-t \lambda \sigma_{n+1}}{2}} P_{\sigma_{n+1}}+\prod_{j=1}^{n+1} P_{\sigma_{j}}^{\perp} .
\end{aligned}
$$

Therefore (54) holds $\forall n \in \mathbb{N}$. From this we deduce that, for every finite set $F$

$$
\prod_{\sigma \in F}\left(e^{\frac{-t \lambda_{\sigma}}{2}} P_{\sigma}+P_{\sigma}^{\perp}\right)=\sum_{\sigma \in F} e^{\frac{-t \lambda_{\sigma}}{2}} P_{\sigma}+\prod_{\sigma \in F} P_{\sigma}^{\perp} .
$$

Passing to the limit we see that (52) holds for every countable set $F$.

Finally the assumption that $(|\sigma\rangle)_{\sigma \in T}$ is an orthonormal basis of $\mathcal{H}_{S}$ implies that

$$
\prod_{\sigma \in T} P_{\sigma}^{\perp}=0
$$

COROLLARY 4 Under the assumptions (8) and (31) if

$$
\lambda_{\text {inf }}^{-}:=\inf \lambda_{\sigma}^{-}>0
$$

then one has,

$$
\forall x \quad \lim _{t \rightarrow+\infty} e^{t S_{2}\left(\lambda^{-}\right)}(x)=0
$$


Proof. From (50), (51) it follows that

$$
\prod_{\sigma} V_{\sigma}^{t}=\prod_{\sigma}\left(e^{-t \lambda_{\sigma}^{-} / 2} P_{\sigma}+P_{\sigma}^{\perp}\right)=\sum_{\sigma} e^{-t \lambda_{\sigma} / 2} P_{\sigma}
$$

Therefore

$$
e^{t S_{2}\left(\lambda^{-}\right)}(x)=\left(\prod_{\sigma} V_{\sigma}^{t}\right) x\left(\prod_{\sigma} V_{\sigma}^{t}\right)
$$

with

$$
\left\|e^{t S_{2}\left(\lambda^{-}\right)}(x)\right\| \leq e^{-t \lambda_{\text {inf }}^{-}\|x\|}
$$

and this proves $(56)$.

COROLLARY 5 It holds

$$
e^{t S_{2}}(1)=\sum_{\sigma} e^{-t \lambda_{\sigma}} P_{\sigma}
$$

Proof.

$$
e^{t S_{2}\left(\lambda^{-}\right)}(1)=\prod_{\sigma}\left(V_{\sigma}^{t}\right)^{2}=\prod_{\sigma}\left(e^{-t \lambda_{\sigma} / 2} P_{\sigma}+P_{\sigma}^{\perp}\right)^{2}=\left(\sum_{\sigma} e^{-t \lambda_{\sigma} / 2} P_{\sigma}\right)^{2}
$$

and the thesis follows from (53).

LEMMA 9 Under the assumption (55) one has

$$
\lim _{t \rightarrow \infty} S_{1}\left(e^{-t \lambda^{-}}\right)=0 .
$$

Proof. From (40) we know that $\lambda^{-}$is a diagonal matrix hence, from (36) it follows that

$$
S_{1}\left(\lambda^{-}\right)=\sum_{\sigma} \lambda_{\sigma}^{-} S_{\sigma \sigma}
$$

Therefore

$$
S_{1}\left(e^{-t \lambda^{-}}\right)=\sum_{\sigma} e^{-t \lambda_{\sigma}^{-}} S_{\sigma \sigma}=e^{-t \lambda_{\mathrm{inf}}^{-}} \sum_{\sigma} e^{-t\left(\lambda_{\sigma}^{-}-\lambda_{\mathrm{inf}}^{-}\right)} S_{\sigma \sigma} .
$$

Now for any $x \in \mathcal{B}_{S}$ and $\xi \in \mathcal{H}_{S}$

$$
\begin{aligned}
\left\|\sum_{\sigma} S_{\sigma \sigma}(x) \xi\right\|^{2} & =\left\|\sum_{\sigma} \sigma(x) P_{\sigma} \xi\right\|^{2}=\sum_{\sigma}\left\|\sigma(x) P_{\sigma} \xi\right\|^{2} \leq \sum_{\sigma}|\sigma(x)| 2\left\|P_{\sigma} \xi\right\|^{2} \\
& \leq\|x\|^{2} \sum_{\sigma}\left\|P_{\sigma} \xi\right\|^{2}=\|x\|^{2}\|\xi\|^{2} .
\end{aligned}
$$

Therefore

$$
\left\|S_{1}\left(e^{-t \lambda^{-}}\right)\right\| \leq e^{-t \lambda_{\text {inf }}^{-}\|x\|}
$$

and from (57) follows. 
LEMMA 10 It holds

$$
S_{1}\left(e^{-t \lambda^{-}}\right)(1)=\sum_{\sigma} e^{-t \lambda_{\sigma}} P_{\sigma}
$$

Proof. Using (23) and (36) we find

$$
S_{1}\left(e^{-t \lambda^{-}}\right)(1)=\sum_{\sigma} e^{-t \lambda_{\sigma}^{-}} S_{\sigma, \sigma}(1)=\sum_{\sigma} e^{-t \lambda_{\sigma}^{-}} P_{\sigma}\langle\sigma, 1 \sigma\rangle=\sum_{\sigma} e^{-t \lambda_{\sigma}^{-}} P_{\sigma} .
$$

LEMMA 11 It holds

$$
S_{1}\left(\gamma^{-}-\lambda^{-}\right)(1)=0
$$

Proof. From (36) we have

$$
\begin{aligned}
S_{1}\left(\gamma^{-}-\lambda^{-}\right)(1) & =\sum_{\sigma, \sigma^{\prime}}\left(\gamma_{\sigma \sigma^{\prime}}-\delta_{\sigma \sigma^{\prime}} \lambda_{\sigma^{\prime}}^{-}\right) S_{\sigma \sigma^{\prime}}(1) \\
& =\sum_{\sigma, \sigma^{\prime}} \gamma_{\sigma \sigma^{\prime}} P_{\sigma}-\sum_{\sigma, \sigma^{\prime}} \delta_{\sigma \sigma^{\prime}} \lambda_{\sigma}^{-} P_{\sigma}=\sum_{\sigma} \lambda_{\sigma}^{-} P_{\sigma}-\sum_{\sigma} \lambda_{\sigma}^{-} P_{\sigma}=0
\end{aligned}
$$

Let us summarize the main result of our analysis in the following result which essentially reduces the study of the quantum generators of this class to the study of a classical triangular generator plus a diagonal one, which is easy.

THEOREM 6 In the generic Fock case (8) and for the class of generators (35) satisfying the additional condition (31) the right hand side of the identity

$$
e^{t L^{-}}(x)=\sum_{\sigma \neq \sigma^{\prime}} e^{-t\left(\lambda_{\sigma}^{-}+\lambda_{\sigma^{\prime}}^{-}\right) / 2}\left\langle\sigma, x \sigma^{\prime}\right\rangle|\sigma\rangle\left\langle\sigma^{\prime}\right|+\sum_{\sigma}\left[\sum_{\sigma^{\prime}}\left(e^{t\left(\gamma^{-}-\lambda^{-}\right)}\right)_{\sigma \sigma^{\prime}} \sigma^{\prime}(x)\right] P_{\sigma}
$$

and where the predual semigroup associated is

$$
\left(e^{t L^{-}}\right)_{*}(\rho)=\sum_{\sigma \neq \sigma^{\prime}} e^{-t\left(\lambda_{\sigma}^{-}+\lambda_{\sigma^{\prime}}^{-}\right) / 2}\left\langle\rho \sigma, \sigma^{\prime}\right\rangle\left|\sigma^{\prime}\right\rangle\langle\sigma|+\sum_{\sigma}\left[\sum_{\sigma^{\prime}}\left(e^{t\left(\gamma^{-}-\lambda^{-}\right)}\right)_{\sigma \sigma^{\prime}} P_{\sigma^{\prime}}\right] \sigma(\rho)
$$

defines a solution of the master equation associated to the generator $L^{-}$. This solution is unique if the generator is bounded.

Proof. Let us observe that

$$
\begin{aligned}
e^{t L^{-}}(x) & =e^{t\left(S_{1}\left(\gamma^{-}\right)+S_{2}\left(\lambda^{-}\right)\right)}(x) \\
& =e^{t S_{2}\left(\lambda^{-}\right)}(x)-S_{1}\left(e^{-t \lambda^{-}}\right)(x)+S_{1}\left(e^{t\left(\gamma^{-}-\lambda^{-}\right)}\right)(x),
\end{aligned}
$$


where $\gamma^{-}$and $\lambda^{-}$are given by (30) and (39), respectively. If $(|\sigma\rangle)$ is an orthonormal basis then

$$
\begin{aligned}
e^{t L^{-}}(x)= & \left(\sum_{\sigma} e^{\frac{-t \lambda \bar{\sigma}}{2}} P_{\sigma}\right) x\left(\sum_{\sigma} e^{\frac{-t \lambda \bar{\sigma}}{2}} P_{\sigma}\right) \\
& +\sum_{\sigma, \sigma^{\prime}}\left(e^{-t \lambda^{-}}\right)_{\sigma \sigma^{\prime}} S_{\sigma \sigma^{\prime}}(x)+\sum_{\sigma, \sigma^{\prime}}\left(e^{t\left(\gamma^{-}-\lambda^{-}\right)}\right)_{\sigma \sigma^{\prime}} S_{\sigma \sigma^{\prime}}(x) \\
= & \sum_{\sigma, \sigma^{\prime}} e^{-t\left(\lambda_{\sigma}^{-}+\lambda_{\sigma^{\prime}}^{-}\right) / 2} P_{\sigma} x P_{\sigma^{\prime}}-\sum_{\sigma} e^{-t \lambda \bar{\sigma}} \sigma(x) P_{\sigma} \\
& +\sum_{\sigma \neq \sigma^{\prime}}\left(e^{t\left(\gamma^{-}-\lambda^{-}\right)}\right)_{\sigma \sigma^{\prime}} \sigma^{\prime}(x) P_{\sigma}+\sum_{\sigma}\left(e^{t\left(\gamma^{-}-\lambda^{-}\right)}\right)_{\sigma \sigma} \sigma(x) P_{\sigma} \\
= & \sum_{\sigma \neq \sigma^{\prime}} e^{-t\left(\lambda_{\sigma}+\lambda_{\sigma^{\prime}}\right) / 2}\left\langle\sigma ; x \sigma^{\prime}\right\rangle|\sigma\rangle\left\langle\sigma^{\prime}\right| \\
& +\sum_{\sigma}\left[\left(e^{-t\left(\gamma^{-}-\lambda^{-}\right)}\right)_{\sigma \sigma} \sigma(x)+\sum_{\left\{\sigma^{\prime} ; \sigma^{\prime} \neq \sigma\right\}}\left(e^{-t\left(\gamma^{-}-\lambda^{-}\right)}\right)_{\sigma \sigma^{\prime}} \sigma^{\prime}(x)\right] P_{\sigma} \\
= & \sum_{\sigma \neq \sigma^{\prime}} e^{-t\left(\lambda_{\sigma}^{-}+\lambda_{\sigma^{\prime}}^{-}\right) / 2}\left\langle\sigma, x \sigma^{\prime}\right\rangle|\sigma\rangle\left\langle\sigma^{\prime}\right|+\sum_{\sigma}\left[\sum_{\sigma^{\prime}}\left(e^{t\left(\gamma^{-}-\lambda^{-}\right)}\right)_{\sigma \sigma^{\prime}} \sigma^{\prime}(x)\right] P_{\sigma} .
\end{aligned}
$$

To find the predual semigroup associated, we use the following relation:

$$
\operatorname{tr}\left(\left(e^{t L^{-}}\right)_{*}(\rho) x\right)=\operatorname{tr}\left(\rho\left(e^{t L^{-}}\right)(x)\right)
$$

when $x \in B(H)$ and $\rho \in \mathcal{F}_{1}(H)$.

\section{Acknowledgment}

The authors want to express their gratitude to Franco Fagnola for several suggestions that allowed them to improve the present paper. In particular it was his remark that, even under condition (31), the generator might be unbounded and therefore, in this case, the solution given by our explicit formula might not be unique. The problem to identify this solution with the minimal one, possibly under additional conditions, is now under investigation.

\section{Bibliography}

[1] L. Accardi, Y. G. Lu and I. Volovich, Quantum Theory and its Stochastic Limit, Springer, 2001.

[2] L. Accardi and K. Imafuku, Control of quantum states by decoherence, Preprint Volterra N. 542, March 2003.

[3] L. Accardi, S. V. Kozyrev, Quantum interacting particle systems, Lectures given at the: Volterra-CIRM International School: Quantum interacting particle systems, Levico Terme, 23-29 September 2000, in: QP-PQ XIV, L. Accardi, F. Fagnola, eds., World Scientific, 2002; Preprint Volterra, N. 231, September 2000. 
[4] C. Bahn, Y. M. Park, Feynman-Kac representation and Markov property of semigroups generated by non-commutative elliptic operators, IDAQP 6, 103 (2003).

[5] R. Carbone, Optimal log-Sobolev inequality and hypercontractivity for positive semigroups on $M_{2}(\mathbb{C})$, Infin. Dimens. Anal. Quantum Probab. Relat. Top. 7, 317 (2004).

[6] A. M. Chebotarev, Lectures on quantum probability, Aportaciones Matematicas, Ser. Textos 14, Mexico 2000.

[7] A. M. Chebotarev, Sufficient conditions for dissipative dynamical semigroups to be conservative, Theoret and Math. Phys. 80, 804 (1989).

[8] A. M. Chebotarev and F. Fagnola, Sufficient conditions for conservativiy of minimal quantum dunamical semigroups, J. Funct. Anal. 153, 382 (1989).

[9] F. Fagnola, R. Rebolledo, Quantum Markov semigroups and their stationary states, Stochastic Analysis and Mathematical Physics II (2003), Birkäuser, 2003, pp. 77-128.

[10] J. Garcia, R. Quezada, Hille-Yosida estimate and non-conservativity criteria for quantum dynamical semigroups, IDAQP 7, 383 (2004). 